\title{
EMPIRICAL SCALING FOR PRESENT OHMIC HEATED TOKAMAKS
}

\section{PLASMA PHYSICS LABORATORY}

\section{MASTER}

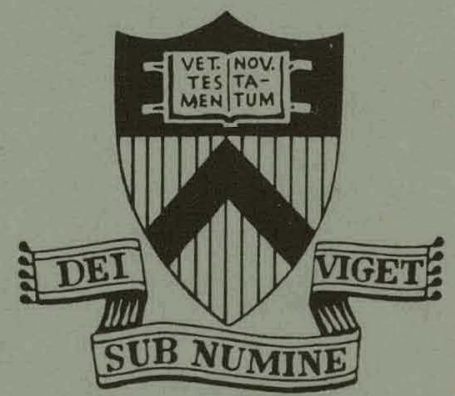

\section{PRINCETON \\ UNIVERSIT Y PRINCETON, NEW JERSEY} This work was supported by U.S. Atomic Energy Commission Contract AT $(11-1)-3073$. Reproduction, transla-
tion, publication, use, and disposal, in whole or in part, by or for the United States Covernment is permitted. 


\section{DISCLAIMER}

This report was prepared as an account of work sponsored by an agency of the United States Government. Neither the United States Government nor any agency Thereof, nor any of their employees, makes any warranty, express or implied, or assumes any legal liability or responsibility for the accuracy, completeness, or usefulness of any information, apparatus, product, or process disclosed, or represents that its use would not infringe privately owned rights. Reference herein to any specific commercial product, process, or service by trade name, trademark, manufacturer, or otherwise does not necessarily constitute or imply its endorsement, recommendation, or favoring by the United States Government or any agency thereof. The views and opinions of authors expressed herein do not necessarily state or reflect those of the United States Government or any agency thereof. 


\section{DISCLAIMER}

Portions of this document may be illegible in electronic image products. Images are produced from the best available original document. 


\title{
EMPIRICAL SCALING FOR PRESENT OHMIC HEATED TOKAMAKS
}

\author{
C. Daughney \\ Princeton University, Plasma Physics Laboratory \\ Princeton, New Jersey 08540
}

\begin{abstract}
Empirical scaling laws are given for the average electron temperature and electron energy confinement time as functions of plasma current, average electron density, effective ion charge, toroidal magnetic field, and major and minor plasma radius. The ohmic heating is classical, and the electron energy transport is anomalous. The present scaling indicates that ohmicheating becomes ineffective with larger experiments.

This repurl was prepared as an accuuml of work
sponsored by the United States Government. Neither
the United States nor the United States Energy
Resparsh and Develnnment Administratinn, nnr any nf
their employees, nor any of their contractors,
subcontractors, or their employees, makes any
warranty, express or implied, or assumes any legal
labully or responsibilty tor the accuracy, completeness
or usefulness of any information, apparatus, product or
process disclosed, or represents that its use would not
itfringe pivately uwned rights.
\end{abstract}




\section{INTRODUCTION}

The construction of the Adiabatic Toroidal Compressor has been described previously, ${ }^{1}$ and experiments on plasma compression, ${ }^{2}$ neutral beam heating ${ }^{3}$, and stability and ohmic heating ${ }^{4}$ have been reported. In this paper, a summary is presented of the electron temperature and density measurements obtained with spectral analysis of Thomson scattered ruby light. In particular, empirical scaling laws are described for the average electron temperature and electron energy confinement time as functions of the plasma current, the average electron density and the effective ion charge. These empirical scaling laws are extended to include dependence upon minor and major plasma radius and toroidal field strength through a comparison of the various Tokamaks described in the literature. $5-9$ Finally, the local energy confinement time for a single discharge is assumed to have the same parametric dependence as that of the average confinement time obtained trom numerous discharges, and it is shown that the predicted radial profile of the electron temperature agrees with the observed profile.

\section{LASER SCATTERING EXPERIMENT}

The experimental arrangement is shown in Figure 1. A Korad K1500 ruby laser is used to give a Q-switched pulse of 3 joules at $.69 \mu$ and approximately $50 \mathrm{nsec}$ halfwidth. The light scattered. from a $1.5 \mathrm{~cm}$ length of the laser beam in the mid plane of the torus is collected over $5 \times 10^{-2}$ sterrad at an angle of $90^{\circ}$. The scattered light is resolved with a $40 \mathrm{~cm}$ Czerny-Turner diffraction 
grating spectrometer into 8 plexiglass light guides which cover approximately $1000 \AA$ on the blue side of the laser wavelength. Light in each channel is detected with an R.C.A. 8853 phototube which is electronically gated for $150 \mathrm{nsec}$. The phototube outputs are integrated, digitized and processed on an I.B.M. 1800 computer.

The phototubes are actually gated twice; once coincident with the laser pulse and once $10 \mu$ sec before the laser pulse in order to monitor the plasma light. Parasitic ruby light is determined on a separate laser shot with no plasma present. The 8 channels are calibrated relatively and absolutely with a tungsten lamp.

\subsection{Computed Quantitiès}

With the spectrometer signals corrected for parasitic scatter, plasma light and integrator zero level as described above, the raw data gives the one-dimensional distribution function for the electrons:

$$
f(\Delta \lambda)=A\left(1-\frac{3}{2} \frac{\Delta \lambda}{\lambda_{\mathrm{R}}}\right) n \exp \left(-\frac{\Delta \lambda}{\lambda_{\odot}}\right)^{2}
$$

where

$$
\begin{aligned}
A & \equiv\left(\frac{m_{e}}{2 \pi e T_{e}}\right)^{1 / 2} \\
\lambda_{0}^{2} & \equiv \frac{8 e \sin ^{2} \frac{\theta}{2}}{m_{e} c^{2}} \lambda_{R}^{2} T_{e}
\end{aligned}
$$

and

$\theta$ is the scattering angle of $90^{\circ}$

$\lambda_{R}$ is the wavelength of the ruby laser 
The factor including $\frac{3}{2} \frac{\Delta \lambda}{\lambda_{\mathrm{R}}}$ is the relativistic correction term which accounts for the relativistic blue shift. This term is in agreement with that used previously, 10 with the important difference that an additional wavelength correction is included due to the fact that a photomultiplier detector is strictly photon sensitive rather than energy sensitive. ${ }^{11}$

A least squares fit was applied to the logarithmic approximation of the distribution function, In $f(\Delta \lambda)$ vs $\Delta \lambda^{2}$, to obtain a first approximation for an iterative least squares fit on the Gaussian distributing function. This fit then gave the electron temperature and density at the given radius and time.

To obtain radial profiles of the electron temperature and density, the laser beam was moved, shot-by-shot, along the major radius. Again a least squares fit was used to fit a trigonometric series to the measured points. The profile was then described by:

$$
P(r)=\sum_{I}^{N} A_{n} \cos \left\{(2 n-1) \frac{\left(R-R_{0}\right)}{a} \frac{\pi}{2}\right\} 1 \leq N \leq 9
$$

Typically three terms were used and the same radius parameter (a) was used to obtain both density and temperature profiles. However, for some discharges, particularly gettered discharges, 12 it was necessary to use $a_{T}<a_{n} \cdot R_{\delta}$ is the major radius of the plasma torus.

From these profiles various quantities were computed, such as : Poloidal $\beta: \beta_{\theta}=\frac{16 \pi^{2}}{\mu_{0} T^{2}} \int_{0}^{a_{T}} n_{e} e^{e T}$ rdr where $\mu_{0}$ is $4 \pi \times 10^{-7}$ Henry $/ \mathrm{m}$. 
Electron Energy Confinement Time :

$$
\begin{aligned}
\tau_{E} & \equiv{\frac{6 \Pi^{2}}{I V}}^{R_{0}} \int_{0}^{a} n_{e} n^{e T} r d x \\
& \equiv \frac{3}{8} \mu_{0}{\frac{R_{0}}{R_{M}}}^{B_{\theta}}
\end{aligned}
$$

- where $R_{M}$ is the measured plasma resistance.

Effective Ion Charge: $\operatorname{zeff} \equiv \frac{\Sigma\left\langle\mathrm{n}_{i} \mathrm{z}_{i}^{2}\right\rangle}{\left\langle\mathrm{n}_{\mathrm{e}}{ }^{>}\right.}$

This is obtained by assuming the plasma resistance is neoclassical $^{13}$ and the average ion charge is independent of radius. An iterative procedure is used to solve for zeff in the implicit relation:

$$
\frac{1}{R_{M}}=\int_{0}^{a_{T}} \frac{n e^{2}:}{\gamma\left(\text { Zeff) } m_{e} \nu_{e i}\right.}\left(i-\frac{1.9\left(\frac{r}{R_{o}}\right)^{1 / 2}}{1+\gamma(\text { Zeff }) \nu}\right) \frac{r}{R_{0}} d r
$$

where $\nu_{e i}$ is the classical electron ion collision frequency

$$
\begin{aligned}
& \gamma(\text { Zeff })=\frac{.46 \text { Zeff }}{1.08+\text { Zeff }}+.29 \text { Zeff } \\
& \nu^{*}=\nu_{\text {ei } q \frac{R_{o}}{v_{e}}\left(\frac{R_{0}}{r}\right)^{3 / 2}} \\
& q .=\frac{2 \pi r^{2} B \phi}{\mu_{0} I(r) R_{0}}
\end{aligned}
$$




$$
v_{e}=\sqrt{\frac{2 e T_{e}}{m_{e}}}
$$

The second term in the bracket above is the neo-classical correction due to trapped electrons which are excluded from carrying current. For all discharges discussed in this paper, the correction was small; amounting to $\leqslant 20 \%$ increase in the plasma resistance compared with the classical value. Since $\nu$ * tends to Infinity for both $r=0$ and $r$ a, an dvelage riblue was computed as:

$$
\left\langle v^{*}\right\rangle=\left[\frac{2}{a^{2} \operatorname{zeff}} \int \frac{r d r}{v^{*}(r)}\right]^{-1}
$$

For the discharges considered $1 \leqslant\langle\nu *\rangle \leqslant 10$.

\section{A.T.C. OPERATION}

All data discussed in this paper was obtained with the plasma in the uncompressed position, i.e. plasma major radius of $.87 \mathrm{~m}$ and minor radius of $.17 \mathrm{~m}$. The minor radius is determined by upper and lower rail limiters manufactured from either molybdenum or stainless steel. The toroidal field was $1.6 \mathrm{~T}$ and the plasma current was varied between 30 and $100 \mathrm{kA}$. The base pressure was approximately $10^{-7}$ torr and the filling pressure was varied between 1 and $7 \times 10^{-5}$ torr of either $\mathrm{H}_{2}$ or $\mathrm{D}_{2}$. (There was no discernable difference in discharge parameters for the two gases.) The initial pressure determined the initial plasma density, but the electron density could then rise, fall, or remain steady with time, depending presumably, upon the condition of the limiters and/or vacuum walls. 
Spectroscopically, the main impurity in the discharge, was oxygen, with some iron, carbon and molybdenum also observed. 4 The effective ion charge, as determined from the measured plasma resistance and electron temperature profile, was typically about 5. This is a factor of two higher than that obtained spectroscopically, but is in good agreement with the value obtained from pitch angle scattering of injected hydrogen. ${ }^{3}$ The effective ion charge could be reduced to 1 in discharges with titanium deposited on the limiters and vacuum wall.12

Typical radial profiles of electron temperature and density are given in Figure 2. These measurements were made at the time of peak current ( $30 \mathrm{msec}$ ). The accuracy of temperature measurement was typically $\pm 10 \%$ and was limited by photon statistics. The accuracy of density measurement was also $\pm 10 \%$ and was limited by reproducibility of the discharge. Figure 2 illustrates the typical profile shapes observed. The temperature profile was reasonably approximated by a $\left(1-[r / a]^{2}\right)^{2}$ dependence. (Discharges with low zeff tended to be contracted in minor radius, i.e. the minor radius used in the profile fit was less than the limiter value). The electron density profile wa's typically broader than the temperature profile and could be approximated by $\left(1-[r / a]^{2}\right)$. However, the electron temperature profile appeared to be independent of the electron density profile. Similar temperatures (peak and profile) have been reported for two discharges in which current, voltage and toroidal field were the same, but the average electron density differed by a factor of two. ${ }^{4}$ In Figures 3,4 , and 5 , two discharges are compared which have similar average densit.y 
(Note line density curve of Fig. 3) but the peak electron density of Figure 5 is $50 \%$ higher than that of Figure 4 . However, the temperature profile is similar in the two cases.

Figure 3 illustrates typical time evolution of A.T.C. discharges. The line density measured with a $4 \mathrm{~mm}$ microwave interferometer shows a fast initial rise which typically corresponds to half the neutral gas filling pressure. From this time, the average density can rise, fall or remain steady with no apparent effect upon the electron temperature. Figures 4 and 5 illustrate that the electron temperature profile is established early, at $1 b-20 \mathrm{msec}$, and thon remains steady for 15-20 $\mathrm{msec}$ until the vertical field decays at $45 \mathrm{msec}$ and plasma equilibrium is lost. Note that the temperature profile can show a strong axial peaking early in the discharge, as in Fig. 4, but this peaking rapidly relaxes to the typical profile shape.

\section{A.T.C. SCALING}

In this section we derive empirical scaling laws from approximately 80 ATC electron temperature and density profiles obtained with the Thomson scattering diagnostic. The range of machine parameters covered are $32<I<108 \mathrm{kA}$, $.8<\overline{\mathrm{n}}_{\mathrm{e}}<3.5 \times 10^{19} \mathrm{~m}^{-3}, 90<\overline{\mathrm{T}}_{\mathrm{e}}<560 \mathrm{eV}$, and $1<$ Zeff $<9$. For all discharges $\mathrm{a}=.17 \mathrm{~m}, \mathrm{R}=.87 \mathrm{~m}$ and $\mathrm{B} \phi=1.6 \mathrm{~T}$.

As with all Tokamak investigations, ${ }^{5-9}$ we find

$$
\beta_{\Theta} \propto<\mathrm{n}^{>}
$$


In Fig. $\left.6 \mathrm{~A}, \mathrm{~B}_{\theta} /<\mathrm{n}_{e}\right\rangle$ is plotted against $I$ for given values of Zeff; and, in Fig. $6 \mathrm{~B}_{,} \beta_{\dot{\theta}} /\left\langle\mathrm{n}_{\mathrm{e}}\right\rangle^{\prime}$ is plotted against Zeff for given I. "The result is

$$
\beta_{\theta} \propto \frac{<n e^{>}}{I^{3 / 2}}(\operatorname{zeff})^{1 / 2}
$$

since $\beta_{\theta}$ is defined as

$$
B_{\theta} \propto \frac{<n e^{><T} e^{>}}{I^{2}}
$$

this gives a relationship between $\left\langle T_{e}\right\rangle, I$ and zeff for an ohmic heated Tokamak:

$$
\left\langle\mathrm{T}_{\mathrm{e}} \mathrm{C}^{\prime} \propto(\text { Zeff } I)^{1 / 2}\right.
$$

Since Zeff is defined as

$$
\text { Zeff } \propto R_{m}<\mathrm{T}_{\mathrm{e}}{ }^{3 / 2}
$$

then dependence 2 above also gives

$$
\left\langle\mathrm{T}^{>} \quad \propto \quad \mathrm{v}^{2}\right.
$$

These dependences of average electron temperature are plotted in Figs. 7 and 8. Figure 7A shows good agreement for $\left\langle\mathrm{T}_{\mathrm{e}}>\right.$ vs. Zeff. ${ }^{1 / 2}$ Figure $7 \mathrm{~B}$ is consistent with $\left\langle\mathrm{T}_{\mathrm{e}}>\propto \mathrm{I}^{1 / 2}\right.$ but a stronger dependence upon $I$ is possible. Figure 8 shows poor agreement for $\left\langle\mathrm{T}_{\mathrm{e}}\right\rangle \propto \mathrm{V}^{2}$. High and low voltages (or temperatures) do not correlate with current, effective ion chapge or electron density. There is a tendency for the low voltage, high temperature 
discharges to have broad profiles and the high voltage, low temperature discharges to have narrow profiles.

It appears that ionization, radiation and charge exchange from recycling particles can introduce an energy loss which causes the plasma column to shrink from the limiter. Relations 4 and 6 should probably include this effective radius, $\left\langle\mathrm{T}_{\mathrm{e}} \propto(\operatorname{Zeff} \mathrm{I})^{1 / 2} / \mathrm{a}_{\mathrm{T}}\right.$ and $\left\langle\mathrm{T}_{\mathrm{e}}\right\rangle \propto \mathrm{V}^{2} \mathrm{a}_{\mathrm{T}}^{4}$.

The empirical parametric dependence of the average electron energy confinement time is given in Fig. 9.

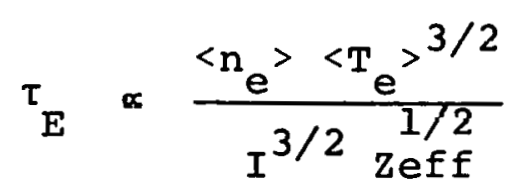

This scaling is very similar to that reported previously 4

$$
\tau_{E} \propto \frac{n_{e} q(0)}{R_{M}} \propto \frac{<n_{e^{>}}<T_{e}>^{3 / 2}}{I Z \text { eff }}
$$

Substituting dependence 4 in 7 to eliminate Zeff, we have:

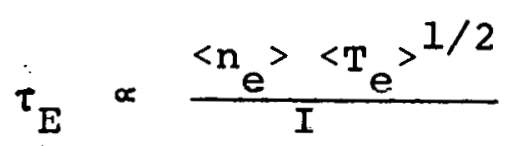

This dependence upon $\left\langle\mathrm{n}_{\mathrm{e}}\right\rangle$ and. I has been reported previously for high density discharges in the T4 machine. 7

Note that the definitions of $\tau_{E}$ ' and $\beta_{\Theta}$ give

$$
R_{M} \propto \frac{B_{\theta}}{\tau_{E}} \propto \frac{\text { Zeff }}{\left\langle T_{e}\right\rangle^{3 / 2}}
$$

This is consistent with our initial assumption that the plasma resistance is classical. 
There is considerable literature on the heat loss from the electrons of an ohmic heated Tokamak. Artsimovitch suggests. there must be an anomalous electron thermal conductivity. ${ }^{14}$ our empirical scaling for this anomalous electron thermal conductivity is

$$
\begin{array}{r}
x_{e} \propto \frac{I^{3 / 2}(\text { Zeff })^{I / 2}}{<n_{\left.e<T_{e}\right)^{3 / 2}}} \\
\text { or } x_{e} \propto \frac{I}{<n_{e}><T_{e}{ }^{I / 2}}
\end{array}
$$

This interpretation of the electron energy confinement time neglects electron radiation losses. This omission is justified in present Tokamaks by recent bolometric measurements on A.T.C. which show the radiated power loss during the steady state of the electron temperature profile is $\lesssim 20 \%$ of the total loss. 4

The density and current dependences are very different from the "pseudo-classical"scaling obtained by Artsimovitch. 14

$$
x_{p c} \propto v_{e i} \rho_{\Theta}^{2} \propto \frac{<n_{e}>\text { Zeff }}{I^{2}<T_{e}>^{l / 2}}
$$

\section{TOKAMAK SCALING}

In this section, an attempt is made to generalize the above scaling laws by comparing Tokamak machines of different major and minor radius and toroidal field strength. A summary of machine parameters found in the literature is given in Table 1. The range of parameters available is $.80<\mathrm{R}_{0}<1.09 \mathrm{~m}$. $.06<\mathrm{a}<.23 \mathrm{~m}$. and $1.6<\mathrm{B} \phi<5.0 \mathrm{~T}$. The list is restricted 
to those papers which give sufficient experimental data for the calculation of zeff.

In Fig. 10, the electron energy confinement time is plotted against minor radius for the machines described in Table I. The temperature and current dependence has been removed by using relation 7 above It is apparent that there is a strong dependence upon minor radius, and weak dependence upon toroidal magnetic field and major radius.

$$
\tau_{E} \propto \frac{\left\langle n_{e}\right\rangle\left\langle T_{e}\right\rangle^{3 / 2}}{\operatorname{li2} I^{3 / 2}} a^{3}
$$

For power balance in an ohmic heated Tokamak with this empirical confinement time, we have

$$
\begin{aligned}
& P_{\text {in }} \equiv I^{2} R_{M}=P_{\text {loss }} \equiv\left\langle n_{e}\right\rangle\left\langle T_{e}\right\rangle 3 \pi^{2} a^{2} R_{o} / \tau_{E}
\end{aligned}
$$

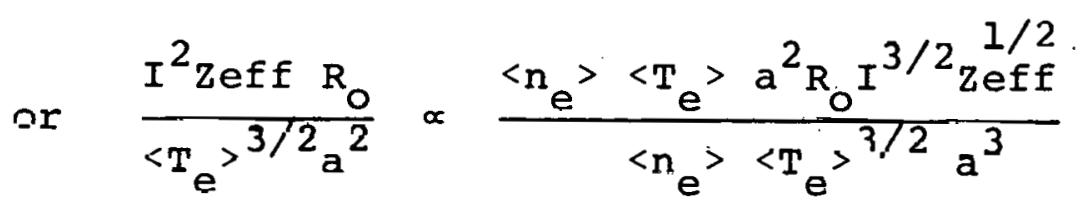

$$
\begin{aligned}
& \text { i.e. } \quad\left\langle T_{e}\right\rangle \propto \frac{(\operatorname{Zeff} I)^{1 / 2}}{a}
\end{aligned}
$$

This dependence is plotted in Fig. 11.

Tt. is apparent that present ohmic heated tokamaks have the same scaling observed for the A.T.C. machine.

$$
\left\langle T_{e}\right\rangle=\frac{(\operatorname{Zeff} I)^{1 / 2}}{10 a}
$$

and

$$
\tau_{E}=\frac{3<\mathrm{n}_{e^{><T} e^{>^{3 / 2}}}}{I^{3 / 2}} \mathrm{a}^{3} \times 10^{-16}
$$


or

$$
\tau_{E}=\frac{3<\mathrm{n}_{e^{><T} e^{>^{1 / 2}}}}{I} a^{2} \cdot 10^{-17}
$$

all in M.K.S. units.

These values are much lower than calculated with the numerical codes using pseudo classical transport coefficients. Furthermore, $\left\langle\mathrm{T}_{\mathrm{e}}\right\rangle$ would be reduced by a factor of approximately 2 in a clean hydrogen discharge and the confinement time reduced accordingly.

\section{A.T.C. PROFILE}

If it is assumed that the local energy confinement time $\tau_{E}(r)$ and thermal conductivity $x_{e}(r)$ have the same scaling as determined from the average quantities. Then the power balance during the steady-state portion of the A.T.C. discharge may be used to predict the radial temperature profile.

$$
\begin{aligned}
& \text { Since } \frac{P_{L}(r)}{2 \pi R_{0}}=-r n_{e}(r) e x_{e}(r) \frac{d T_{e}(r)}{d r}=\frac{V I(r)}{2 \pi R_{O}} \\
& \text { and } x_{e}=\frac{a^{2}}{\tau_{E}(r)}
\end{aligned}
$$

where $\tau_{E}(r)=3 \times 10^{-17} \frac{n_{e}(r) T_{e}{ }^{1 / 2}(r)}{I(r)} r^{2}$

$$
\text { then } \frac{d T e^{(r)}}{T_{e}^{1 / 2}}=35 \mathrm{~V} \times \frac{d r}{a^{2}}
$$$$
\text { or. } T_{e}=80 \mathrm{v}^{2}\left(1-\frac{\mathrm{r}^{2}}{\mathrm{a}^{2}}\right)^{2},\left\langle\mathrm{~T}_{\mathrm{e}}\right\rangle=\frac{80 \mathrm{v}^{2}}{3}
$$ 
This computed electron temperature profile is in agreement with the observed profiles shown in Figs. 2, 4 and 5. The computed average electron temperature scales with voltage as suggested above and is in agreement with the mean value of. $<\mathrm{T}_{\mathrm{e}}>$ vs. $\mathrm{V}^{2}$ given in Fig. 8 .

\section{DISCUSSION}

The values of zeff determined from resistance measurement, spectroscopic analysis and fast ion scattering times are all in agreement. ${ }^{3}$ Furthermore, with titanium gettering, zeff can be reduced to 1 in a hydrogen discharge and to 2 in a helium discharge. ${ }^{12}$ This suggests that the plasma resistance is classical. It is however, necessary to consider the effective ion charge due to impurity ions in the discharge. In present tokamaks, the plasma resistance is enhanced by a factor of 1-10 over that of a hydrogenic plasma. The heat loss from an ohmic heated tokamak is anomalous. In the A.T.C. tokamak, the particle confinement time is approximately an order of magnitude longer than the electron energy confinement time, and this implies the anomalous heat loss results from an enhanced electron thermal conductivity. Theoretical considerations suggest the trapped electron mode is most likely to cause the enhanced thermal conductivity in present tokamaks. ${ }^{15}$. The results obtained here agree in magnitude, but do not agree in parametric dependence.

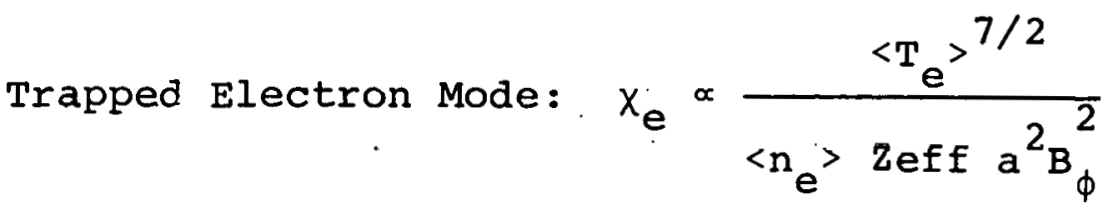


Empirical Result: $\quad x_{e} \propto \frac{I^{3 / 2}(\text { Zeff) })^{1 / 2}}{\left\langle n_{e}>\left\langle T_{e}\right\rangle^{3 / 2} a\right.}$

If the same transport process occurs in a larger ohmic heated tokamak as is observed in present tokamaks, then the electron temperature and confinement time can be estimated. The parameters of the P.I.T. device are:

$$
\begin{array}{ll}
I=1 . \mathrm{M} . \mathrm{A} . & \mathrm{a}=.45 \mathrm{~m} \\
B_{\phi}=4 \mathrm{~T} . & \mathrm{R}_{\mathrm{O}}=1.30 \mathrm{~m}
\end{array}
$$

Note that $B_{\phi}$ and $R_{0}$ are not changed appreciably from present tokamaks. From Figs. 10 and 11, we have:

$$
\begin{aligned}
\left\langle\mathrm{T}_{\mathrm{e}}\right\rangle & \sim \frac{(\text { Zeff) }}{4.5} \times 10^{3} \mathrm{eV} \\
& \sim 450 \mathrm{eV} \text { for } \operatorname{Zeff}=4 \ldots \\
\text { Peak } \mathrm{T}_{\mathrm{e}} & \sim 1400 \mathrm{eV} \\
\tau_{\mathrm{E}} & \sim 13<\mathrm{n} \mathrm{e}^{>} \times 10^{-23} \mathrm{sec} \\
& \sim 13 \mathrm{msec} \text { for }<\mathrm{n}^{>} \sim 10^{20} \mathrm{~m}^{-3}
\end{aligned}
$$


Numerical simulation codes for tokamaks, ${ }^{16}$ which use an electron thermal conductivity enhanced by the trapped electron mode, predict a peak electron temperature of approximately $2000 \mathrm{eV}$ and an energy confinement time of approximately $100 \mathrm{~ms}$. The numerical codes include enhanced transport in the plasma core to simulate the M.H.D. activity which has been observed experimentally with X-ray techniques. 17 The numerical codes also include loss effects due to recycling particles from the walls and ilmiters. These effecte are ignored in the present analysis. Given the plasma minor radius, which if different from the limiter radius must result from a balance of ohmic power input and losses due to recycling at the plasma edge, and given the safety factor; $q_{a}$, is greater than two; then the electron thermal conductivity is assumed to dominate the entire profile.

\section{CONCLUSION}

Observation of power balance during the steady state phase of the A.T.C. discharge has given empirical scaling for the electron energy confinement time in terms of the average electron: temperature and density, the effective ion charge and lhe plasma current. This empirical scaling is confirmed with data from other tokamaks.

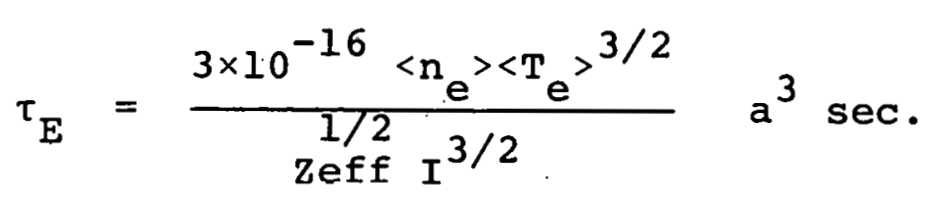


The range of values for major radius and toroidal magnetic field was small and further dependence upon these parameters is possible. Furthermore, due to the interdependence of $\tau_{E}$ ' I and $<\mathrm{T}_{\mathrm{e}}>$ in an ohmic heated tokamak, the above expression has not been proved unique. For example,

$$
\tau_{E}=\frac{3 \times 10^{-17}<n_{e^{><T}} e^{1 / 2}}{I} \cdot a^{2} \mathrm{sec} .
$$

describes the radial power balance in A.T.C. (see section 5), and is consistent with the above expression, given that:

$$
\left\langle\mathrm{T}_{\mathrm{e}}{ }=\frac{(\text { Zeff I })^{1 / 2}}{10 \mathrm{a}} \mathrm{eV}\right.
$$

This final relation was confirmed both for A.T.C. data over the ranges $1 \leq$ zeff $\leq 9$ and $30 \leq I \leq 100 \mathrm{kA}$ and for three other tokamaks over the range $.06 \leq a \leq .23 \mathrm{~m}$.

Empirically, the electron temperature is proportional to the square root of the current density for ohmic heating. Since the current density will not change appreciably in future devices due to limits on the safety factor and toroidal magnetic field, then it is apparent that large powers of auxiliary heating (of order IMW for P.L.T.) must be used to raise the average electron temperature above the present value of about $1000 \mathrm{eV}$. 


\section{ACKNOWLEDGEMENTS}

Both the A.T.C. machine and the Thomson scattering system were operating successfully when the author joined the Princeton Plasma Physics Laboratory. The assistance of R. E. Ellis, K. Bol, and R. Shoemaker of the A.T.C. group and D. L. Dimock, E. Mazzucato and E. Tolnas of the spectroscopy group is particularly appreciated. Helpful discussions with J. A. Schmidt are gratefully acknowledged. This work was supported by United Stated Energy Research and Development Administration Contract E(11-1)-3073. 
TABLE I .

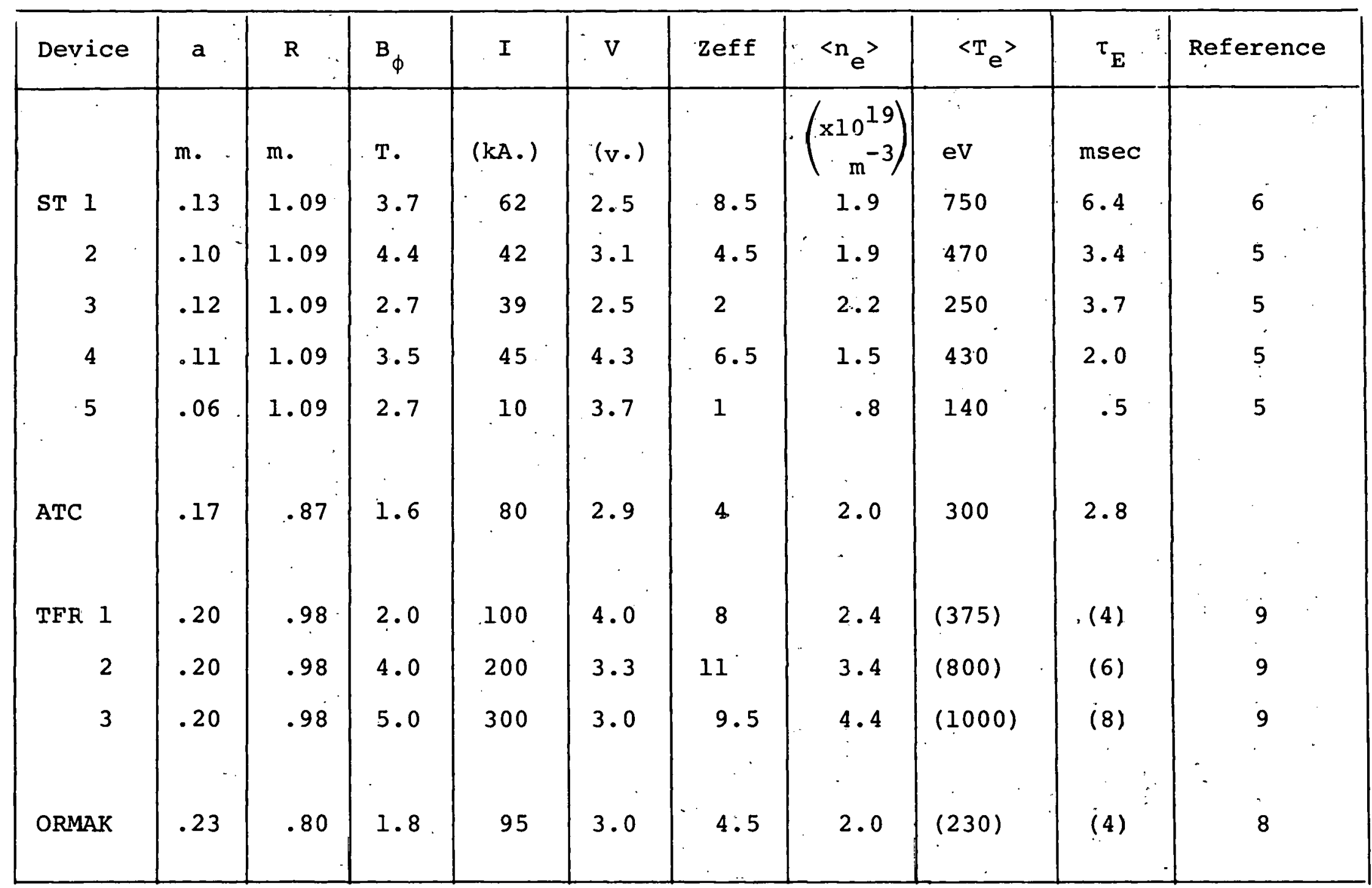




\section{REFERENCES}

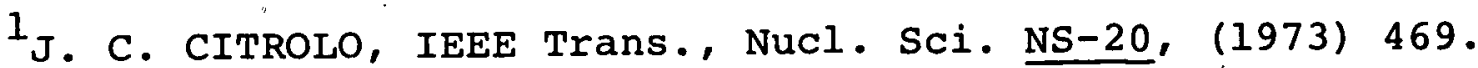
N. M. TURITZIN, 5th Symp. on Eng. Prob. of Fus. Res., (1974) 550. ${ }^{2}$ K. BOL, ET AL, Phys. Rev. Lett. 29, (Nov. 1972) 1495.

${ }^{3}$ K. BOL, ET AL, P1. Phys, and cont. Nuc. Research (IAEA, Tokyo, 1974) to be published, Paper A4-1.

${ }^{4}$ K. BOL, ET AL, Pl. Phys. and Cont. Nuc. Research (IAEA, Tokyo, 1974) to be published, Paper A4-2.

$5_{D}$. DIMOCK, ET $\Lambda L, P l$. Phys. and Cnnt.. Nuc. Fus. Research 1 , (IAEA Vienna, 1971) 451.

${ }^{6}$ D. DIMOCK, ET AL, Nucl. Fus. 13, (1973) 271.

${ }^{7}$ V. A. VERSHKOV, ET AL, Kurchatov Inst. Atomic Energy, Moscow (1973), Trans: PPL-M.T. 112.

8. A. BERRY, ET AL, Pl. Phys, and Cont. Nuc. Research (IAEA, Tokyo, 1974) to be published, Paper A5-1.

9 T.F.R. GROUP, Pl. Phys. and Cont. Nuc. Research (IAEA, Tokyo, 1974) to be published, Paper A6-2.

10 A. M. GONDHALEKAR AND B. KRONAST, Phys. Rev. A $\underline{8}$, (July, 1973) 441 .

${ }^{11}$ D: DIMOCK, private communication.

12P. E. STOTT, ET AL, Princeton Plasma Physics Laboratory Rep. MATT-1111, (Jan, 1975) .

13 F. L. HINTON, ET AL, Phys. Rev. Lett. 29, (Sept. 1972) 698. 
${ }^{14}$ L: A. ARTSIMOVITCH, Nucl. Fus. 12, (1972) 215.

15 B. B. KADOMTSEV AND O. P. POGUTSE, Nucl. Fus. 11 , (Jan., 1971) 67 .

${ }^{16}$ D. F. DÜCHS, ET AL, PI. Phys, and Cont. Nuc. Fus. Research 1, (IAEA, Vienna, 1971) 369 .

17. VON GOELER, ET AL, PhYS. Rev. Lett. 33, (NOv. 1974) 1201. 


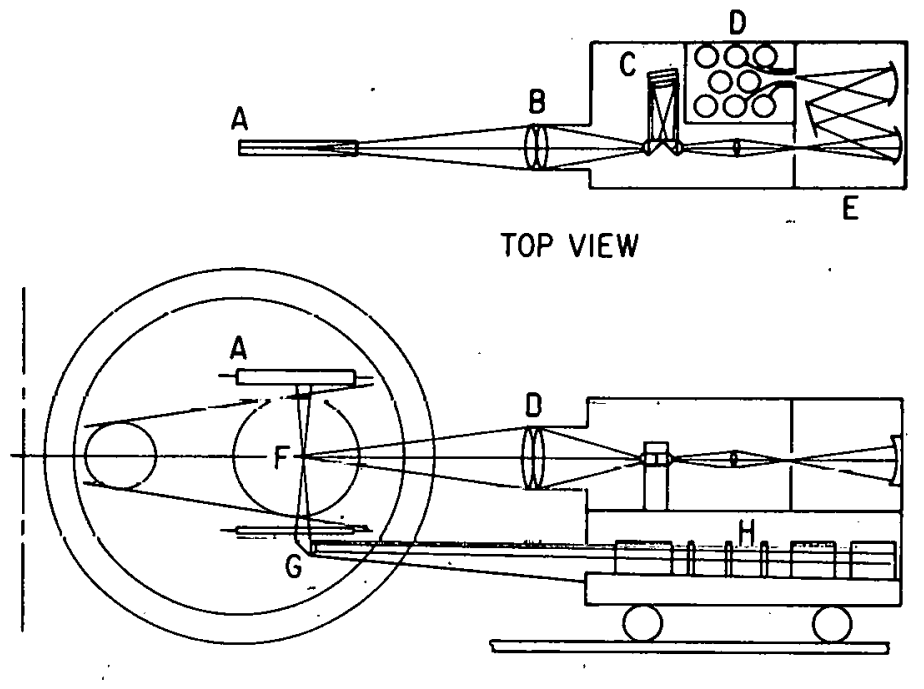

ATC DEVICE

MOVABLE LASER CARRIAGE

SIDE VIEW

Fig. 1. Schematic drawing of Thomson scattering experiment. (H) Q-switched ruby laser, (G) Movable lens and prism below fixed vacuum window, (F) Laser focal point and image of spectrometer slit, A.T.C. uncompressed plasma position, (A) Black glass laser beam dump, (B) Collecting lens, (C) Filter and black glass cutting off ruby and longer wavelength, (E) Grating spectrometer and (D) Light pipe and photomultiplier housing. 


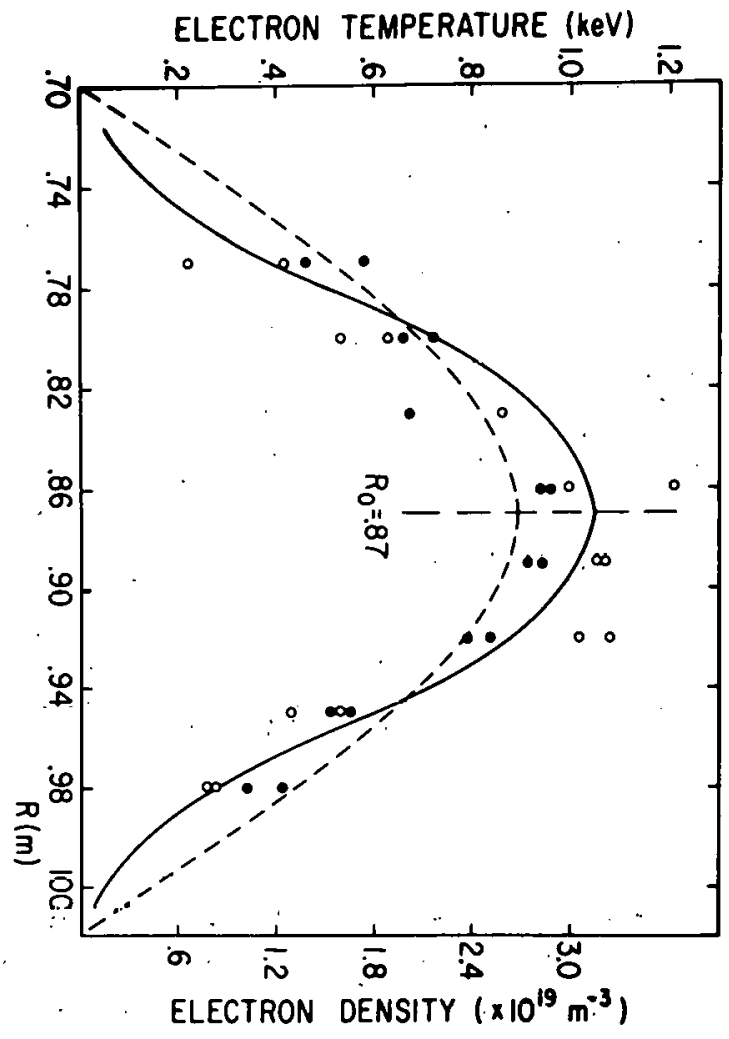

Fig. 2. Electron temperature (open circle) and electron density (closed circles) as functions of major radius, çuřves give typical A.T.C. profiles, $\mathrm{T}_{\mathrm{e}}=\mathrm{T}_{\text {eo }}$ $\left(1-r_{2}^{2} / a_{2}^{2}\right)^{2}$ (solid line) and $n_{e}=n_{\text {eo }}{ }_{1050 \mathrm{eV}}$, $\left(1-r / a^{2}\right)$
$n_{e o}=2.7 \times 10$

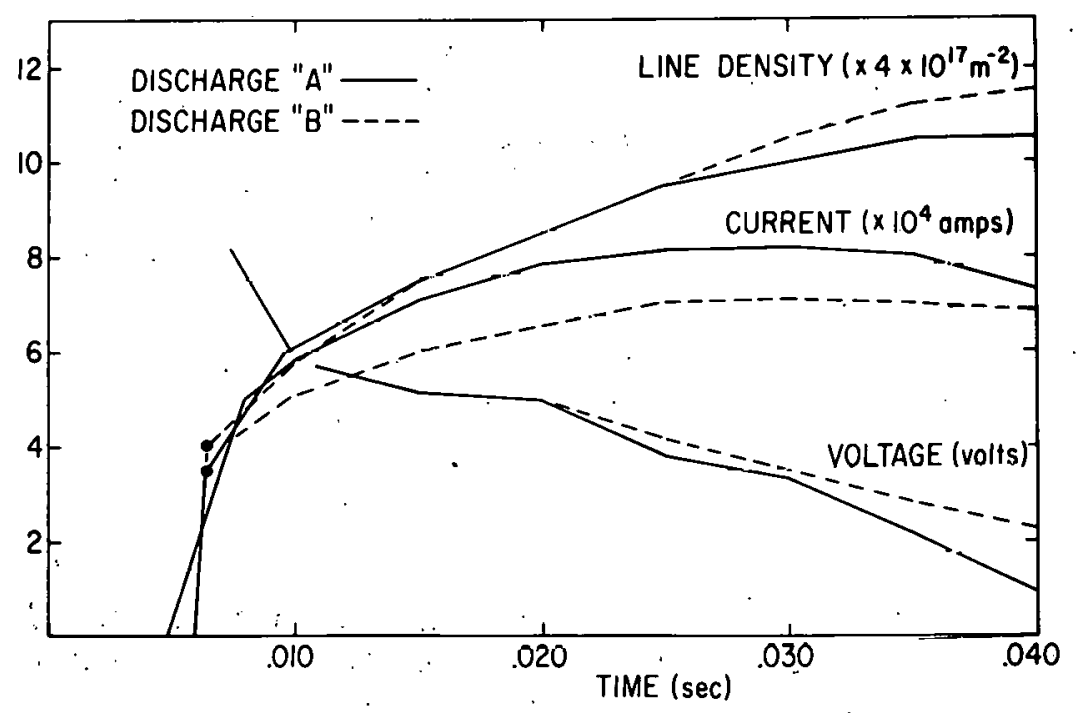

753177

Fig. 3. Plasma current, loop voltage, and line density (4mm. microwave signal) as functions of time. Note that plasma density riscs duriny pulse from initial values given by closed circles. 


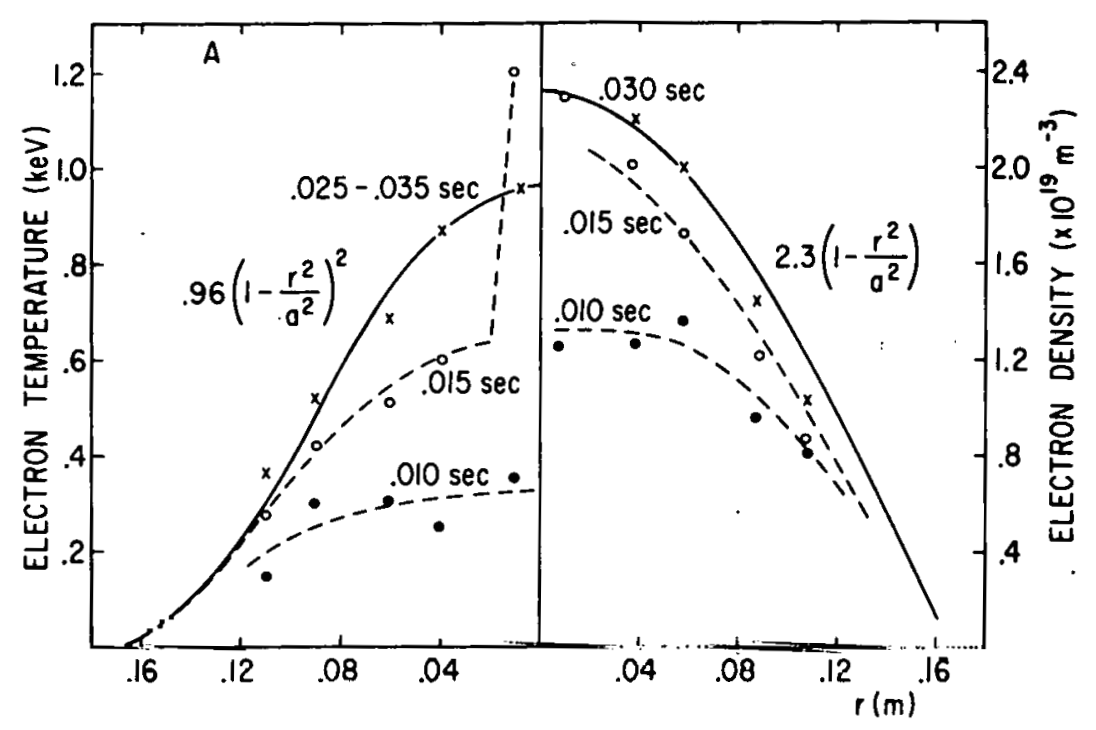

75.3179

Fig. 4. Discharge A. Electron temperature (left) and density (right) as functions of minor radius at time .010,.015 and .030 seconds (see F1g. 3).

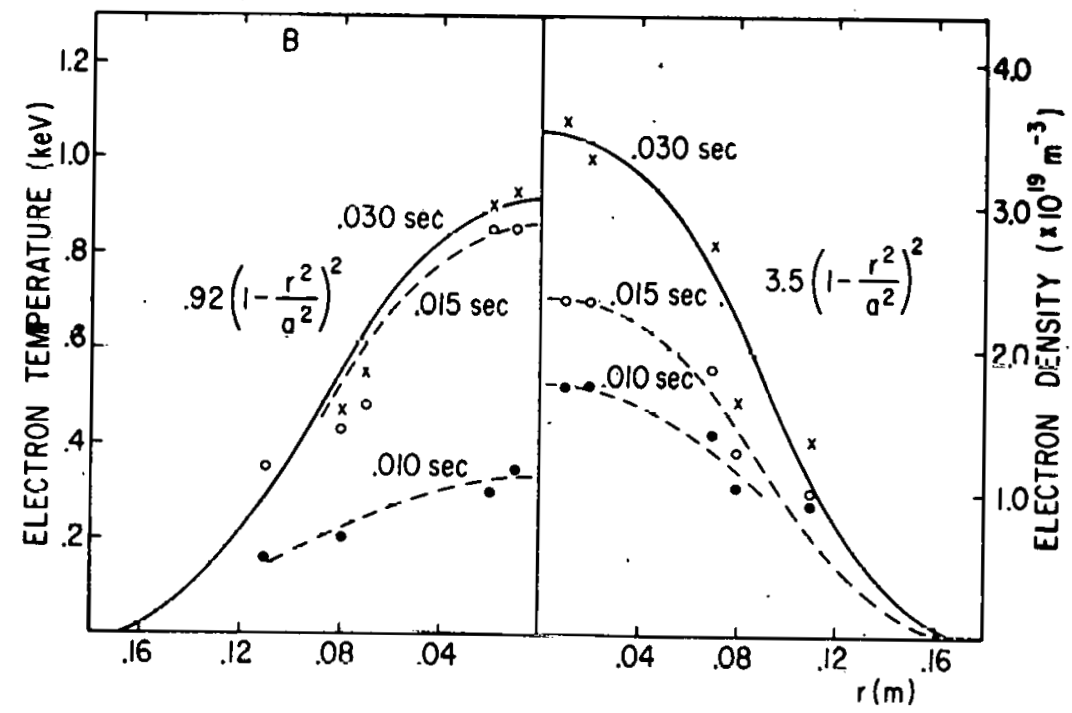

153178

Fig. 5, Discharge B. Electron temperature (left) and density (right) as functions of minor radius at times .010,.015 and .030 seconds (see Fig. 3). 


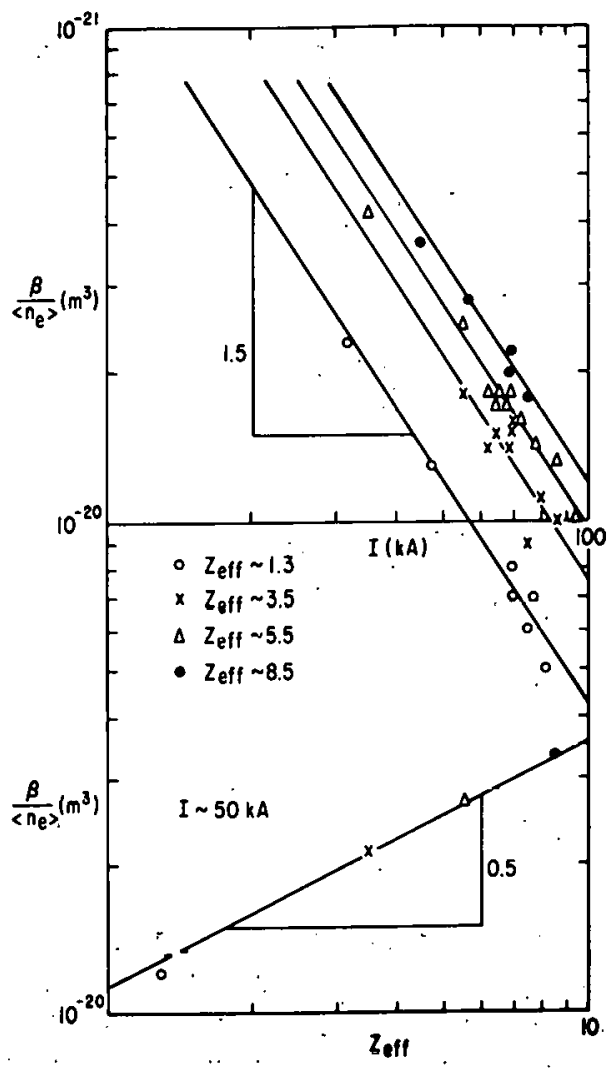

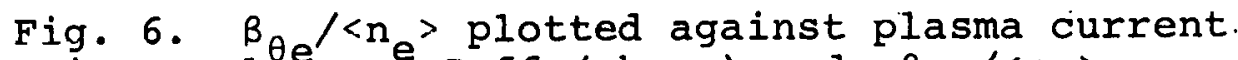
for various values of zeff (above) and $\beta_{\theta e^{/<n}} e^{>}$ plotted against zeff for 39 jven plasma current (below) i.e. $\beta_{\theta} \propto<n e^{>\operatorname{zeff}} 172 / I^{392}$.

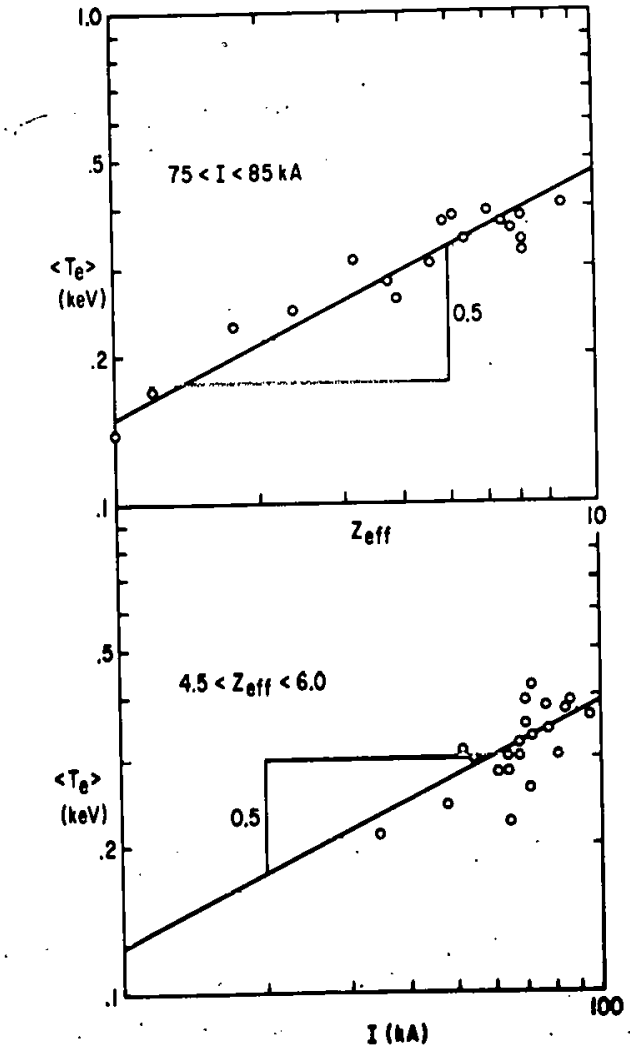

Fig. 7. Average electron temperature plotted against zeff for given plasma cürrent (above) and average electron teinperature against plasma current for given zeff (below). 


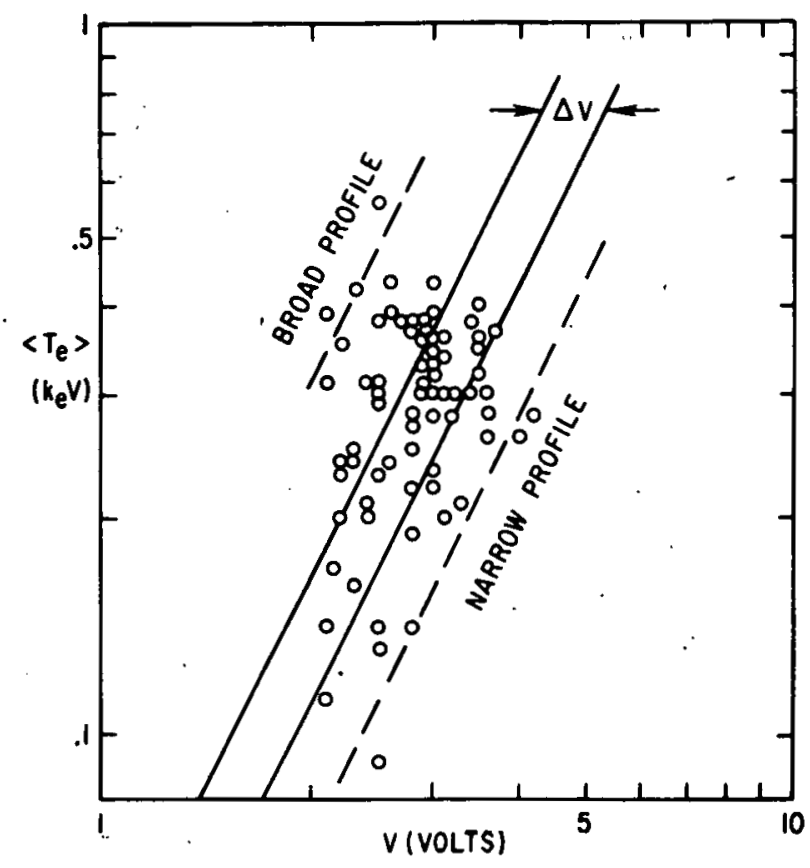

Fig. 8. Average electron temperature plotted against plasma loop voltage. $\Delta \mathrm{V}$ represents uncertainty of voltage measurement due to noise on voltage signal. The minor radius of the electron temperature profile varied from . $11-.17 \mathrm{~m}$.

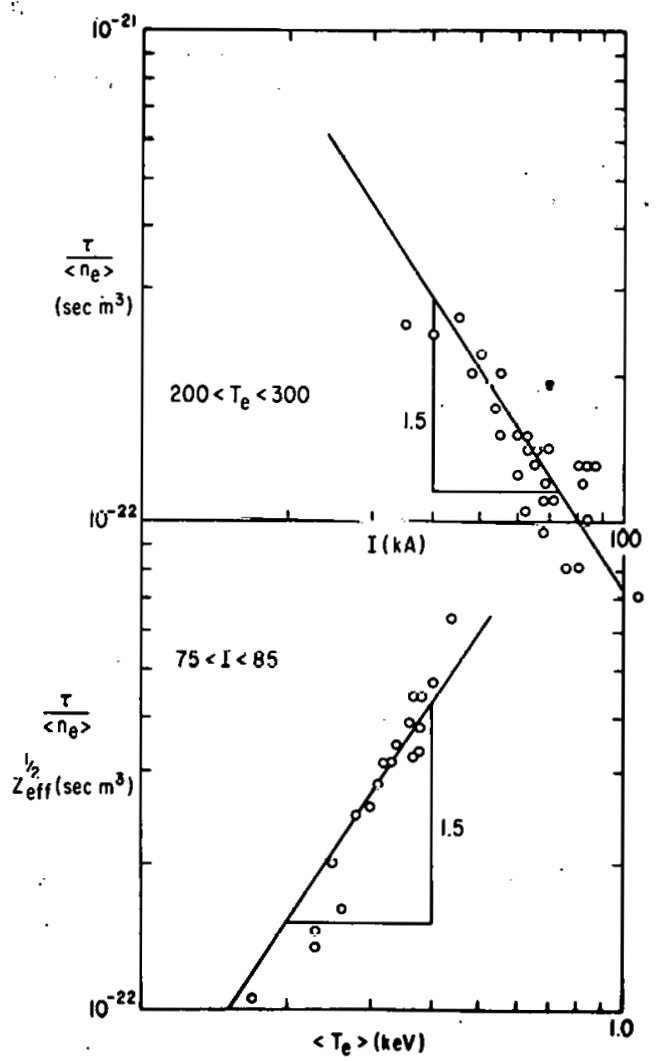

753181

Fig. 9. Scaling of electron energy confinement time $(\tau) . \tau /<n e^{>}$against plasma current for given average lectron temperature (above) and $\tau \cdot \operatorname{Zeff} I / 2 /<n_{\text {e }}>$ against average electron temperature for given plasma currept $($ below) i.e.

$\tau \propto<\mathrm{n}_{\mathrm{e}}><\mathrm{T}_{\mathrm{e}^{>3 / 2} / \mathrm{Zeff} \mathrm{I} / 2} \mathrm{I}^{3} 2$. 


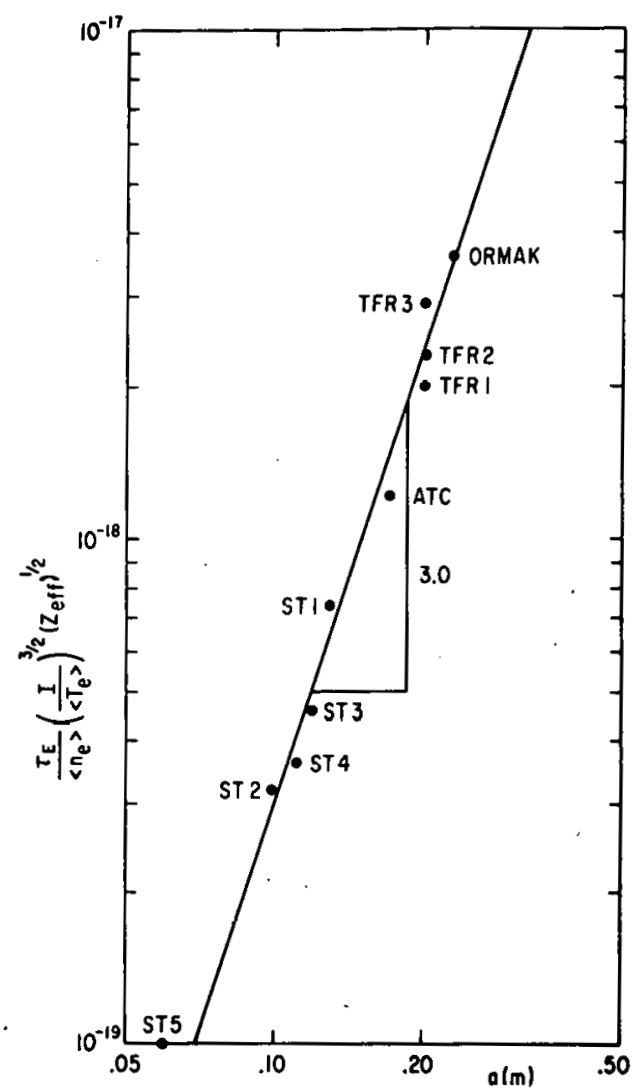

753317

Fig. 10. Normalized electron energy confinement time plotted against plasma minor radius for various tokamak devices identified in Table I. $\tau_{E}=3 \times 10^{-16}$ $<\mathrm{n}_{\mathrm{e}}><\mathrm{T}_{\mathrm{e}}>3 / 2 /$ zeff $\mathrm{I} / 2\left(\mathrm{a}^{2} / \mathrm{I}\right)^{3 / 2}$.

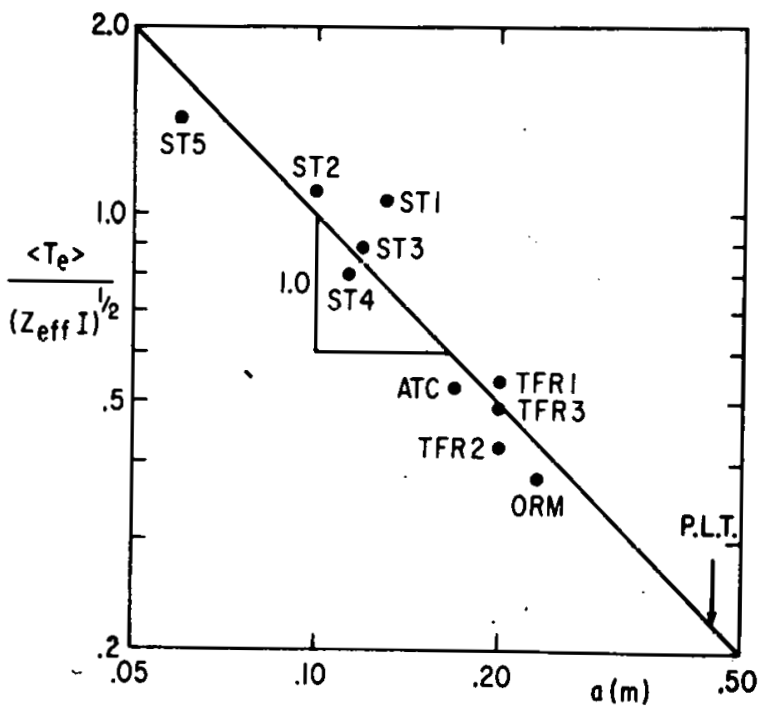

753316

Fig. 11. Normalized electron temperature plotted against plasma minor radius for various tokamak devices identified in Table I. Note projected value for P.L.T. device. $\left\langle\mathrm{T}_{\mathrm{e}}\right\rangle=\left(\right.$ Zeff $\left.\mathrm{I} / 100 \mathrm{a}^{2}\right) 172$. 
NOTICE

This report was prepared as an account of work sponsored by the United States Government. Neither the United States nor the United States Atomic Energy Commission, nor any of their employees, nor any of their contractors, subcontractors, or their employees, makes any warranty, express or implied, or assumes any legal liability or responsibility for the accuracy, completeness or usefulness of any information, apparatus, product or process disclosed, or represents that its use would not infringe privately owned rights. 